\title{
Öko-Audit für die Landwirtschaft?
}

\author{
Die Landwirtschaft ist wie kaum ein anderer Wirtschaftszweig von der Umwelt \\ abhängig. Der Einbezug der landwirtschaftlichen Betriebe in das Öko-Audit-Sy- \\ stem liegt daher nahe, greift aber zu kurz. Die aktuelle Diskussion auch auf eu- \\ ropäischer Ebene geht in Richtung einer Kopplung von Öko-Audit und agrarpoli- \\ tischen Fördermaßnahmen.
}

M Öko-Audit seit März 2001 auch für die Landwirtschaft geöffnet. Ob und in welchem Ausmaß sich das Agrar-Öko-Audit durchsetzen wird, ist allerdings fraglich. Da das Öko-Audit ein freiwilliges Instrument ist, wird jeder Betrieb das Kosten-Nutzen-Verhältnis, das sich aus seiner Durchführung ergibt, abwägen. Die einzelbetriebliche Vorteilhaftigkeit kann aber durch eine Verknüpfung eines Audits mit anderen agrar-, umwelt- und regionalpolitischen Maßnahmen gesteigert werden. Bereits seit längerem wird diskutiert, inwieweit ein AgrarÖko-Audit durch diese Maßnahmen gefördert werden kann und soll, ob die Existenz eines betrieblichen Öko-Audits zur Voraussetzung für Beihilfen an die Landwirtschaft erhoben, bzw. ob die Höhe der Förderung an die Ergebnisse eines solchen Audits (Umweltverträglichkeit des Betriebes) gebunden werden soll (1). Erst kürzlich hat EU Agrarkommissar Fischler im Rahmen seiner Überlegungen zur Halbzeitbewertung der Agenda 2000 einen ähnlichen Vorschlag, nämlich die Einfiuhrung eines so genannten Farm Audits, unterbreitet.

\section{- Freiwilliges Agrar-Öko-Audit?}

In den letzten Jahren haben sich einzelne, meist größere landwirtschaftliche Betriebe zur Einführung eines Agrar-Öko-Audits entschlossen. Dabei handelt es sich jedoch vorwiegend um Pilotvorhaben, die zudem von den - meist östlichen - Bundesländern finanziell unterstützt wurden. Eine größere Verbreitung auf freiwilliger Basis ist jedoch nicht erwartbar.

Einmal ist zu sehen, dass die zusätzliche Kostenund Arbeitsbelastung durch eine Umweltbetriebsprüfung und den Aufbau eines Umweltmanagementsystems erheblich sind. Besonders bei kleinen landwirtschaftlichen Familienbetrieben dürfte vielfach der Nutzen, den ein Öko-Audit-Siegel für sie bringt, kaum die Kosten- und Arbeitsbelastungen aufwiegen. Das Rationalisierungspotenzial ist bei einem Großteil der landwirtschaftlichen Betriebe aufgrund des hohen Kosteneinsparungsdrucks bereits weitgehend ausgereizt. Auch eine Erzielung von Preisvorteilen aufgrund eines Öko-Audit-Siegels ist bei dem Großteil der Betriebe, die homogene Massenprodukte, wie zum Beispiel Getreide, Zuckerrüben oder Milch an Großbetriebe der Ernährungsindustrie liefern, nicht realistisch.

Landwirtschaftliche Betriebe allerdings, die öffentlichkeitsnah wirtschaften und mit Direktvermarktung oder Bereitstellung von Urlaubsangeboten den Verbraucher direkt erreichen, können schon jetzt einen Marketingvorteil vom Agrar-Öko-Audit erwarten. Darüber hinaus kann durch Druck der Verarbeitungsunternehmen, des Handels und der Öffentlichkeit eine gewisse Ausweitung des ÖkoAudits in der Landwirtschaft erreicht werden. Auf diesem Wege könnte das durch die Lebensmittelskandale der letzten Jahre erschütterte Vertrauen der Verbraucher zurückgewonnen werden.

Abgesehen von diesen Einzelbeispielen wird für einen Großteil der Landwirtschaft aus den oben genannten Gründen nur nach einer einschneidenden Veränderung der Begleitumstände die Durchführung eines Öko-Audit attraktiv werden.

\section{Das Agrar-Öko-Audit als Teil eines Instrumentenmixes}

Um die breite Masse von Betrieben zu erreichen, könnte eine Kopplung des Audits an Subventionen und Fördermaßnahmen erfolgen. In diese Richtung geht auch Fischlers Farm-Audit. Es soll für alle Haupterwerbsbetriebe mit Direktzahlungen von mehr als 5000 Euro pro Jahr - dies entspricht etwa einem 20 Hektar Betrieb - als Voraussetzung für eine weitere Gewährung der Zahlungen dienen. Ein Teil der Kosten für die Durchführung des Audits soll durch die Umschichtung finanzieller Mittel aus der Agrarpreisstïtzung finanziert werden. Nähere
Details über Inhalt, Organisation und Ablauf des Farm Audits sind bislang noch nicht bekannt. Vermutlich handelt es sich dabei ,nur" um ein vereinfachtes Pflichtaudit, das im Wesentlichen die Grundsätze der ,guten fachlichen Praxis“ abprüft. Nach den bislang bekannt gewordenen Kommissionsvorstellungen ist alleine die Durchführung eines Audits Voraussetzung für den Erhalt von Beihilfen, nicht aber das Ausmaß der tatsächlichen Umweltverbesserung.

Dies könnte durch eine Verknüpfung des ÖkoAudit mit einer gezielten Honorierung ökologischer Leistungen erreicht werden (2). Dabei würden die bisher nicht an Umweltkriterien gebundenen Flächen- und Tierprämien durch leistungsorientierte Zahlungen für öffentliche Güter ersetzt. Ein Punktesystem, das auf Stoffbilanzen, Bewirtschaftungstechniken und Agrarlandschaftsindikatoren basiert, würde eine Orientierungshilfe für die Honorierungssätze geben.

Mit einer solch tiefgreifenden Modifikation des Öko-Audits würde allerdings das Freiwilligkeitsprinzip, das ein wichtiger Bestandteil der Öko-Audit-Idee ist, vollständig aufgegeben. In der jetzigen Situation der Landwirtschaft kann jedoch mit einem unverbindlichen Instrument, das für den größten Teil der Betriebe kaum einen wirtschaftlichen Nutzen bringt, nicht viel erreicht werden. Die Bindung des Öko-Audits an Förderungen stellt hingegen ein durchschlagendes Mittel dar, das zu einem Umdenken in der Landwirtschaft führen kann.

\section{Anmerkungen}

(1) Vgl. z.B. Bauer, S./ Abresch, J.P./ Spindler, E.: Hammer Thesen zum Agrar-Öko-Audit: Neue Perspektiven für eine umwelthewusste Gartenbau- und Landwirtschaft. In: Spindler, E. (Hrsg.): Agrar-Öko-Audit: Praxis und Perspektiven einer umweltorientierten Land- und Forstwirtschaft, S. 7-12.

(2) Vgl. hierzu ausführlich Bauer, S.: Agrar-Öko-Audit und Honorierung ökologischer Leistungen. In: Spindler E. (Hrsg.): AgrarÖko-Audit - Agrarwende mit System, Ergebnisse eines Workshops im Auftrag des Bundesministeriums für Umwelt, Naturschutz und Reaktorsicherheit, Frankfurt 2002, S. 118-129.

\section{Die Autorlnnen}

Dr. Siegfried Bauer ist Professor für Projekt- und Regionalplanung an der Universität Gießen. Vanessa Andreadakis ist dort wissenschaftliche Mitarbeiterin. Kontakt: Fachbereich Agrarwissenschaften, Ökotrophologie und Umweltmanagement an der JustusLiebig-Universität Gießen, Senckenbergstr. 3, 35390 Gießen. Tel. 0641-99373-10, Fax -19, E-Mail: Siegfried.Bauer@agrar.uni-giessen.de 
(c) 20I0 Authors; licensee IÖW and oekom verlag. This is an article distributed under the terms of the Creative Commons Attribution Non-Commercial No Derivates License (http://creativecommons.org/licenses/by-nc-nd/3.o/), which permits unrestricted use, distribution, and reproduction in any medium, provided the original work is properly cited. 